\title{
TEST PLAN FOR A CALIBRATION DEMONSTRATION SYSTEM FOR THE REFLECTED SOLAR INSTRUMENT FOR THE CLIMATE ABSOLUTE RADIANCE AND REFRACTIVITY OBSERVATORY
}

\author{
Kurtis Thome, Joel McCorkel, Jason Hair, Brendan McAndrew, Adrian Daw, Donald Jennings, \\ Douglas Rabin
}

\author{
NASA, Goddard Space Flight Center
}

The Climate Absolute Radiance and Refractivity Observatory (CLARREO) mission addresses the need to observe high-accuracy, long-term climate change trends and to use decadal change observations as the most critical method to determine the accuracy of climate change. One of the major objectives of CLARREO is to advance the accuracy of SI traceable absolute calibration at infrared and reflected solar wavelengths. This advance is required to reach the on-orbit absolute accuracy required to allow climate change observations to survive data gaps while remaining sufficiently accurate to observe climate change to within the uncertainty of the limit of natural variability. While these capabilities exist at NIST in the laboratory, there is a need to demonstrate that it can move successfully from NIST to NASA and/or instrument vendor capabilities for future spaceborne instruments.

The current work describes the test plan for the Solar, Lunar for Absolute Reflectance Imaging Spectroradiometer (SOLARIS) which is the calibration demonstration system (CDS) for the reflected solar portion of CLARREO. The goal of the CDS is to allow the testing and evaluation of calibration approaches, alternate design and/or implementation approaches and components for the CLARREO mission. SOLARIS also provides a test-bed for detector technologies, non-linearity determination and uncertainties, and application of future technology developments and suggested spacecraft instrument design modifications. The end result of efforts with the SOLARIS CDS will be an SI-traceable error budget for reflectance retrieval using solar irradiance as a reference and methods for laboratory-based, absolute calibration suitable for climate-quality data collections. 
The CLARREO mission addresses the need to observe high-accuracy, long-term climate change trends and advance the accuracy of SI traceable absolute calibration. The current work describes the test plan for the SOLARIS which is the calibration demonstration system for the reflected solar portion of CLARREO. SOLARIS provides a test-bed for detector technologies, non-linearity determination and uncertainties, and application of future technology developments and suggested spacecraft instrument design modifications. The end result will be an SI-traceable error budget for reflectance retrieval using solar irradiance as a reference and methods for laboratory-based, absolute calibration suitable for climate-quality data collections. 